\title{
Next Generation Digital Commerce Technologies
}

\author{
doi:10.3991/ijim.v3i2.655
}

Dr. Muzhir Shaban Al-Ani

Amman Arab University of Graduate Studies, Amman, Jordan

\begin{abstract}
This paper deals with the demonstration of commerce technologies and concentration on the next generation technologies. The development of high advance technologies in telecommunications such as internet and mobile telephony leads to massive support for digital commerce. The main feature of the third generation mobile internet protocol addressing introduced an access to the internet through mobile. The trends of next generation digital commerce try to overcome all the commercial problems and to develop a fast secure and intelligent commerce depending on the high performance next generation technologies.
\end{abstract}

Index Terms-Next Generation Commerce, Next Generation Internet, Next Generation Mobile, Next Generation Commerce Technologies.

\section{INTRODUCTION}

The first efficient communication system for long distance was the post letters system; this was established from the beginning of civilization with different manners. This system was advanced by introducing new technologies in transferring and distributing letters and documentations through cities and countries, but this system suffer from disturbance with the environment effects and also it is not fast. Recently many rules and protocols were established between countries to explain and manage this system.

Marconi invented the wireless telegraph 1896, and in 1901 he sent telegraphic signals across the Atlantic Ocean. His invention allowed two parties to communicate by sending each other alphanumeric characters, this leads to a big revolution in wireless communication systems [1].

The origin of telecommunications and wireless networks starts with the first radio transmission in 1896. Few years after, the invention of telephone was occurred. In 1901 Marconi successfully transmitted a radio signal across the Atlantic Ocean and in 1902 the first bidirectional communication across the Atlantic Ocean was established. The origins of radio based telephony dated back to 1915, when the first radio based conversation was established between ships to transfer information [1].

In 1946, the first public Mobile Telephone System (MTS) was introduced in 25 cities in the USA. Due to this old technology that the mobile transceiver was so big therefore it was used for car-based mobile telephony. MTS was analog system and half duplex transmission. MTS was improved in 1960, to put in operation a full duplex Improved Mobile Telephone System (IMTS). This leads to the cellular concept of transmission [2].

Cellular Mobile communication system passing from analog to digital forms and leads a huge revolution of telecommunications worldwide. In other word this field becomes the future of the world in investment, advertising, business, commerce, marketing, transaction, management, learning, health and other activities of human life as shown in figure 1.

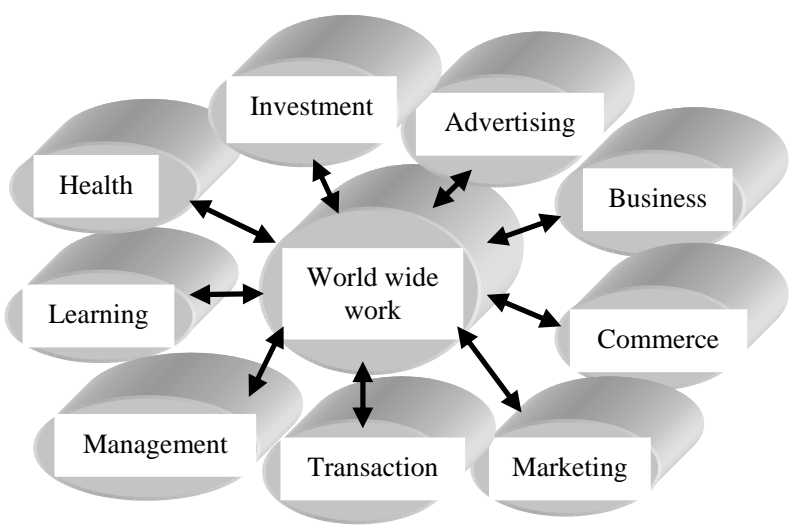

Figure 1. Worldwide work

\section{INTERNET TECHNOLOGIES}

The introduction of electronic computers in the mid1950s coincided with a tremendous boom in economic development, which raised the level of data storage and retrieval requirement. In 1981, International Business Machines Corporation (IBM) introduced the IBM personal computers (PCs), making computing legitimate in business, industry and organizations. These PCs could be linked together in computer networks to be powerful to timeshare several users. Recently manufacturing of PCs are more advanced and use high technologies which raise speed and capacity.

In the late 1960s, the Advanced Research Project Agency (ARPA) of the department of defense was established as a first network between universities and research institution with communication lines operating at 56kbps. That was the beginning of the ARPAnet and allowed for multiple users to communicate simultaneously over the same communication paths [2\&3].

The protocol for communicating over the ARPAnet became known as Transmission Control Protocol (TCP) which ensured that messages were properly routed from sender to receiver. A wide variety of networking hardware and software appeared. ARPA accomplished their work with the development of Internetworking protocol (IP). The combined set of protocols is now called TCP/IP. The internet evolved, organizations worldwide were implementing their own networks for both intraorganization (within the organization) and interorganization (between organizations) [2\&3]. 
Companies are tacking advantage of the connectivity and ease of use of internet technology to create internal corporate networks called intranets that are based on internet technology. Private intranets extended to authorized users outside the organization are called extranets, and firms use such networks to coordinate their activities with other firms for marketing, purchases, collaborating on design, and other interorganizational work [4].

The web designer must take various issues into consideration:

- Speed: Consumers may be impressed by a fancy site, or may lack confidence in a firm that offers a simple one. Yet, fancier sites with extensive graphics take time to download.

- Keeping users on the site: A large number of baskets are abandoned online as consumers fail to complete the check-out process for the products they have selected. One problem here is that many consumers are drawn away from a site and then are unlikely to come back. A large number of links may be desirable to consumers, but they tend to draw people away.

- Information collection: An increasing number of consumers resist collection of information about them, and a number of consumers have set up their browsers to disallow cookies, files that contain information about their computers and shopping habits.

- The internet is creating a new universal technology platform on which to build all sorts, of new products, services, information, strategies, and organizations and also the internet offers new business models. Companies can use the internet capabilities to exchange business transactions and all kinds of messages as described in figure 2 [5\&6].

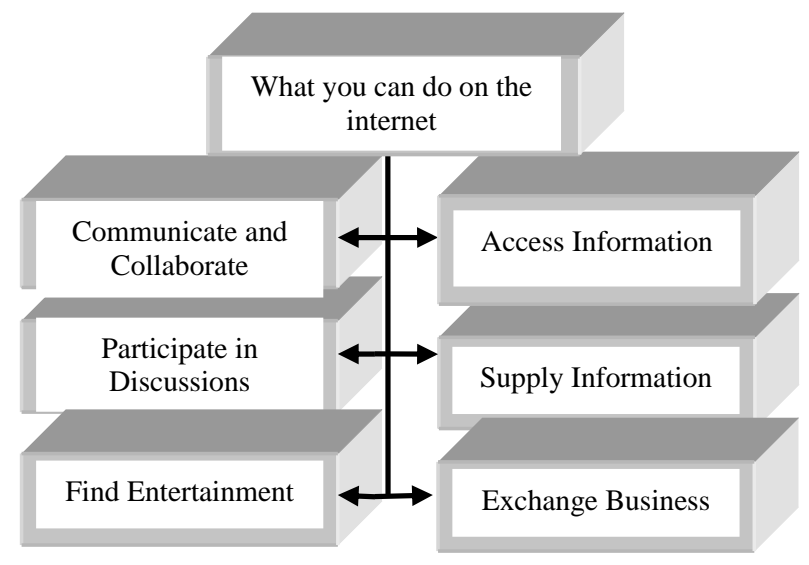

Figure 2. What you can do on the internet

\section{E-COMMERCE}

The growth of the internet, the globalization of trade, the advanced communication systems, challenges the internet technology in supplying the foundation of new business model, new business process, and new ways of marketing and distributing product, services and knowledge.

Electronic commerce (E-commerce) can be establish by connection of commercial systems with the internet by offering products, services and information globally via the Internet. Doing transactions and communications without borders and delays have extensive effects on all branches. E-commerce realizes electronic presentation of products and services, online acceptance of orders, automatic processing of customer inquiries and online payment and transaction processing.

E-commerce is a new term for an old practice. This practice was standardized as Electronic Data Interchange (EDI). EDI is used by businesses to perform billing, ordering, and inventory tracking functions using industryspecific protocols to communicate the necessary information between companies over private, value-added networks. Though EDI has been used strictly to handle commerce between companies, the strong penetration of the Internet and PC into the household and workplace has made electronic commerce between companies and the individual consumer a reality. Although the current level of this type of commerce is small in comparison to the business commerce, it has experienced rapid growth [7\&8].

Online marketing can serve several purposes: Actual sales of products, Promotion/advertising: Customer service, Market research.

There are many obstacles to the growth of e-commerce:

- Reach: Although the majority of U.S. households now have computers connected to the Internet, a minority does not, and penetration rates are considerably lower in some countries.

- Concerns about privacy: A number of consumers are concerned about giving up information to marketers that can easily be collected electronically.

- Reputational issues: Firms operating online or through direct mail have often been viewed with suspicion since consumers may question whether they will be around if they do not deliver satisfactorily.

- Costs: The most successful e-commerce firms turn out to be the ones that have been successful doing other kinds of direct marketing before and have developed the discipline and efficiency required there.

- Language: Since the Internet reaches around the world, it is often difficult to match viewers with their preferred languages. Because U.S. firms and individuals tended to predominate among those first to occupy the Web, most sites are in U.S. English. It is estimated that at 2007, the majority of web surfers will not be comfortable in English but in their own languages.

- Government regulations: In the U.S., the government has tried to keep its hands off the Net as much as possible to foster its growth as a trade area, and a recently expired moratorium on new sales taxes was even instituted to make Internet shopping more attractive. However, governments in many other countries are more forceful in their regulations.

- Cultural obstacles: The whole purpose of the web is to make information readily available. In countries where information is closely guarded, that is a frightening idea. There is often also a desire for personal interaction, which may be required to establish the trust needed to secure a deal.

- Payment issues: U.S. consumers exposed to credit card fraud have very limited liabilities, but these 
protections do not exist to the same extent in Europe or Asia.

If you plan to run your own storefront on the Web, you need more than just your merchant account. You must work with your ISP (Internet Service Provide) to establish SSL (Secure Socket Layer) connections, which will almost always cost more than your standard fees. You also will want to get a payment remittance service on board to transmit the credit card information directly to your bank. If you are already retailing and have a POS terminal, your bank may have package deals available to make this easier.

\section{Mobile TeChNOLOGIES}

First Mobile Telephone System (MTS) was introduced in 1946, but this type of technology was advanced in features and services. Years later was started the cellular telephony. Mobile cellular system can be categorized into different technologies [2\&3]:

First Generation (1G) Cellular Systems: This analog system started in the late 1970 and brought out revolution in the area of mobile telephony and business with some restrictions on encryption, quality, and spectrum efficiency. The first commercial analog system in USA, known as Advanced Mobile Phone System (AMPS), established in 1982, offered only voice transmission. AMPS have also been deployed in other countries. In European countries, several 1G systems have been deployed. The most popular systems are Total Access Communications System (TACS) and Nordic Mobile Telephone (NMT) that covered $50 \%$ of the subscribers in 1995.

Second Generation (2G) Cellular Systems: This system is also called digital system; its activities in Europe were initiated in 1982 by Group Special Mobile that was later renamed to Global System for Mobile Communications (GSM). The first commercial deployment of GSM was made in 1992, known as DCS 1800 that applied in USA. Later 2G became most popular and in 1999 it had one million new subscribers every week. 2G offered more advantages such as voice quality, rooming, saving power, encryption to provide privacy, General Packet Radio Services (GPRS), including support for message services as SMS and support of new services.

Third Generation (3G) Cellular Systems: 3G systems offered global mobile multimedia communication capabilities in an efficient manner. The first recommended for 3G network were made back in 1992, when the internet was still a tool for the academic and technical society. Standardization for 3G system was initiated by the International Telecommunication Union (ITU) in 1992 to establish the International Mobile Telecommunications 2000 (IMT-2000). 3G provided efficient support for both voice and high bit rate data services. 3G services combined telephony, the internet and multimedia services into a single device. 3G having the advantage of support for Internet Protocol (IP) address and enhance mobility. This was a big boom of 3G that permitted it to penetrate deeply the commercial services through Mobile commerce (m-commerce) applications.

Forth Generation (4G) Cellular Systems: As in all areas of technology, the quest for better and more efficient systems never ends and as soon as the time for deployment of a system comes, research on the 4G (also named Next Generation) is usually under way and will reach deployment stage sometime around 2010. It is expected that the evolution of wireless networks towards an integrated system will produce a common packet switched platform for wireless systems, thus enabling the wireless internet.

\section{M-COMMERCE}

M-commerce will introduce flexibility to E-commerce. As most people keep their handsets with them at all time, they will have the ability to make on-line purchases and reservations upon demand without having to be in front of an internet connected PC. Introducing M-commerce to the mobile platform will be an important source of operator revenue.

The technology used to implement GPRS is similar to that used in the Internet. In conventional circuit-based technologies, making a phone call requires establishment of a link or circuit between two points on a line. This circuit is maintained continuously for the duration of the call. In contrast, during a data transfer operation on the Internet, no circuit is established. Data are sent in packets which are tagged with an address to direct them along the correct path. At the destination, all these packets are reassembled in the proper order.

According to [7 \& 8] M-commerce can introduce many applications such as: Mobile broking facilities like automatic alerts for price changes, and transaction of shares. Mobile banking services that promise to allow customers to keep track of their finances at the touch of a button. Content such as news, sports and stock quotes. Services like travel reservations, ticketing, ordering gifts and comparison shopping. Mobile inquiry about availability of services in locality. Mobile-based entertainment services such as music/video. Mobile messaging and mobile chatting.

The reasons behind the success of this M-Commerce ventures lies in its unique features such as:

- The ability to use the service "anywhere and anytime" is seen as its major attraction.

- The attractiveness of the "I-mode" service is assisted by the wide diversity of its contents. I-mode provides direct access to four categories of services: on-line transaction, databases, information, and entertainment.

- Users can also access hundreds of independently launched unlinked "I-mode" sites by simply entering a site's URL on the browser screen.

- Since I-mode is based on packet data transmission technology, users are charged only for how much information they retrieve and actual volume of data exchanged, not the amount of time spent online and spent to access the network.

- The "I-mode" mobile platform has been designed to put the needs of users first and is therefore highly convenient and simple.

- A particularly important function for business and a great convenience for the consumer is the billing system.

Some feature and restrictions of $\mathrm{M}$-commerce will explained as below: 


\section{A. Advantages of M-Commerce}

- The benefits of M-Commerce include customer satisfaction, cost savings, and new business opportunities.

- Use M-Commerce anytime, anywhere with the lightweighted device

- Single owner has control over data whereas the mobile device can be highly personalized.

- M-Commerce can bring the buyer and seller together more easily and facilitate greater profits and a closer customer relationship.

\section{B. Disadvantages of M-Commerce}

- Mobile devices do not generally offer the graphics or processing power of a PC.

- The small screens of mobile devices limit the complexity of applications.

- Each network has a differing approach to MCommerce meaning that the international reach and ubiquity of e-commerce will not be matched.

\section{NG-COMMERCE}

Financial Institutions such as banks see mobile commerce as offering new channels of service to customers as well as offering them new and innovative products. These financial institutions are working to design and implement new applications that will offer mobile payment and mobile brokering. The travel industry, in realizing the possible benefits of $\mathrm{m}$ commerce, is working on technologies that will take care of travel arrangements, update customers on flight status, notify them when this information changes and will offer to make new arrangements based on preset user preferences requiring no input from the user. Therefore, a customer's entire trip can be scheduled and maintained using only their mobile device. The retail sector is also looking into the possibility of using mobile commerce for making the purchase of merchandize easier. Customers will be able to browse and order products while using a cheaper more secure payment method. An example of this is; instead of using paper catalogues, retailers can send customers a list of products that the customer would be interested in, directly to their mobile device. Additionally, retailers will also be able to track customers at all times and notify them of discounts at local stores in which that customer would be interested in. Shopping will also be easier. Soon, phones will be equipped with "bar-code scanners" and shoppers could scan an item and find out its pricing and availability. In the entertainment industry, mcommerce could be used for the purchasing of movie tickets, verify someone's ID or authorize their reservation information [9\&10].

The network was designed to operate without centralized control. Initially, it was limited in use to universities, research centers, military and special organizations. Years later, businesses rapidly realized that they could tune their operations and offer new and better services to their clients, so they started spending vast amount of money to develop and enhance the internet.

In the last decade, consumers use phones and faxes to conduct business. Now E-mail messages, Personal Digital Assistants (PDA), and Web sites on the internet are available. We are becoming increasingly more comfortable with electronic media to conduct all kinds of transactions. This make new technologies part of our daily lives and this is the principle of self-server economy. The consumer population is becoming more and more demanding. Each day many of choice and transactions of spending money are applied. The consumer needs many features that are illustrated in figure 3 . There are different ways that consumers can electronically access information, such as directions to specific address in the real world, these ways are denoted in figure 4 .

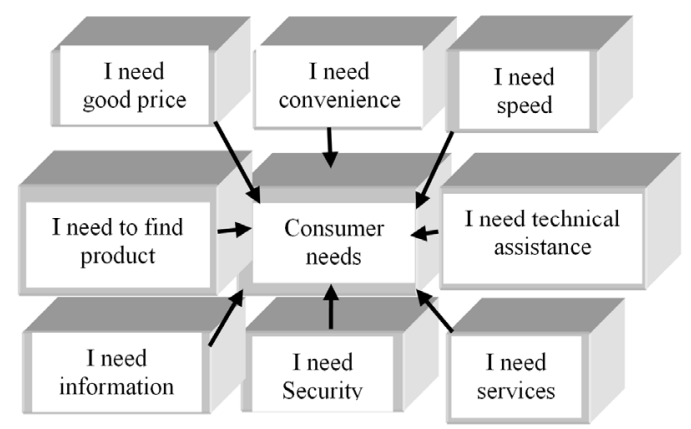

Figure 3. The consumer needs

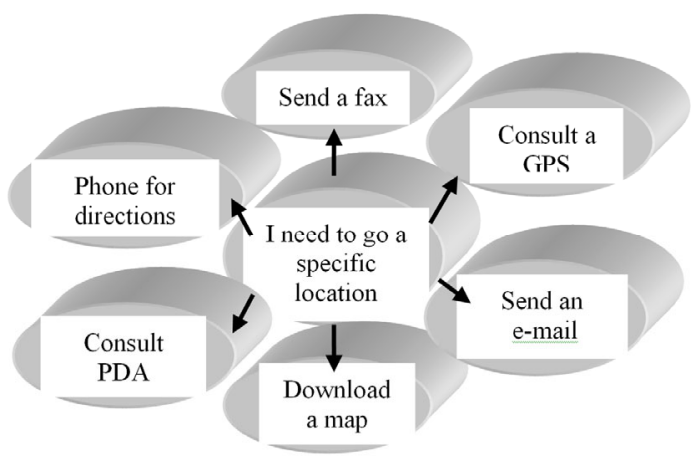

Figure 4. Access mechanisms

The suggested system can be performed by introducing high speed of data transferring through mobile and computer networks to cover the multimedia with an acceptable efficiency. The suggested system include multimedia transmission that suggest the connection between Integrated Service Digital Network (ISDN), the broadband internet (including intranet and extranet) and wireless networks (computer wireless networks and cellular mobile networks). The network of the expected marketing is illustrated in figure 5 in which all these environments are integrated together.

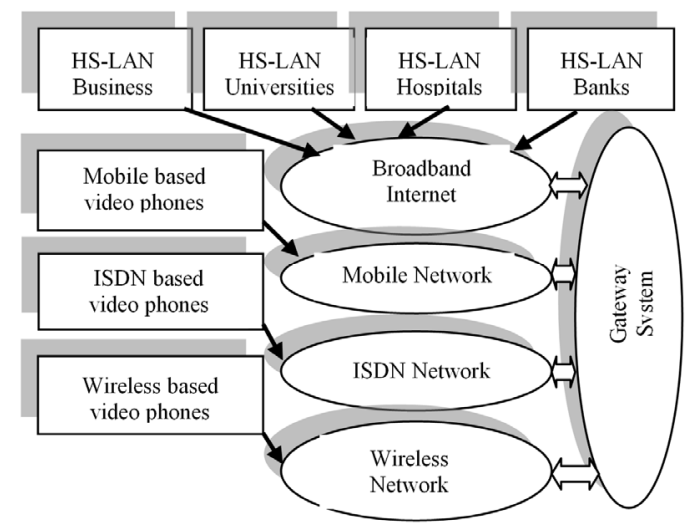

Figure 5. Expected Marketing 


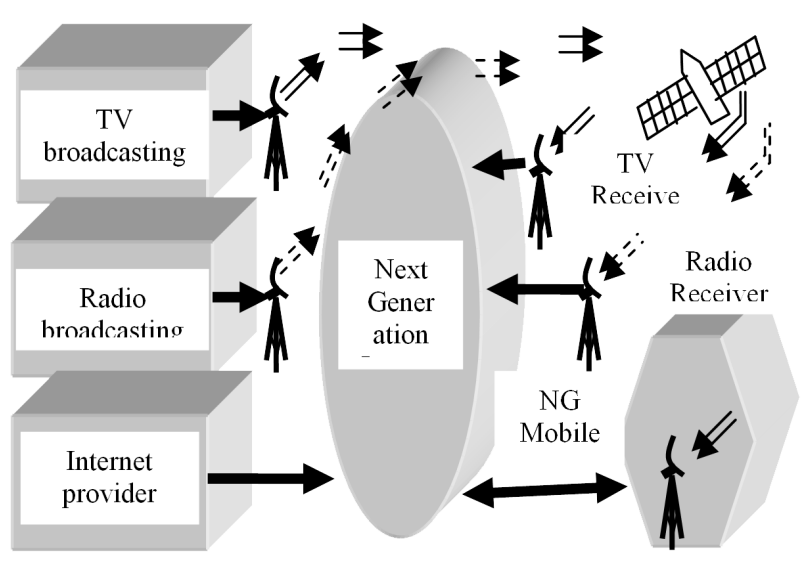

Figure 6. Next Generation Technologies Support Business Multimedia

The suggested system is illustrated in figure 6 that includes of new technologies supported to computers, communications and networking to standardize and merge all forms of data including audio and video in a multimedia streams is imperative. The satellite provider multiplexes a stream of data together with other digital TV channels and transmits it to the customer via satellite. The customer can receive the information with the help of the adapter inside a multimedia PC. This information can be encrypted to ensure that the information is received by the indicated customer without any missing.

\section{CONCLUSIONS}

This paper deals with the demonstration of the advance and future technologies that serve for E-commerce and $\mathrm{M}$ commerce. Many problems will appear through the integration of different multimedia technologies. The framework model presented in this work can help as a general guideline for the next generation digital commerce technologies. The suggested system will use the feature of speed technology of transmission (100 Mbps). This system has the capability to operate on real-time multimedia technologies. The customer can access services and do any interested transaction work without need to any wired connection to the internet. The globalization of industry, business, marketing, information and technologies lead to this integrity of commerce. The new system supports specific features such as high speed, standard, intelligent, secure and simple.

\section{REFERENCES}

[1] M. L. Gillenson, "Fundamentals of Database Management System", John Willy \& Sons, 2005.

[2] W. Stallings, "Wireless Communications and Networks", Prentice Hall 2002.

[3] P. Nicopolitidis \& others, "Wireless Networks", John Wiley \& Sons 2003.

[4] H. M. Deitel and others, Internet \& World Wide Web, Prentice Hall 2002.

[5] K. C. Laudon \&J. P. Laudon, "Management Information System", Pearson Prentice Hall 2004.

[6] P. S. Pressman, "Software Engineering", McGraw Hall 2005.

[7] M. Kodama, "New knowledge creation through leadership based strategic community", Technovation 25, pp. 895-908, Aug. 2005.

[8] C. Maitland \& others, "The European market for mobile data", Tel. Policy 26, pp. 535-554, 2002.

[9] F. Palmieri, " An MPLS based architecture for scalable QoS and Traffic Engineering in Coverage Multiserver Mobile IP Networks", Computer Networks, 47, 2005.

[10] W. H. Melody, "Next Generation Internet", Telecommunication Policy, 24, 2000.

\section{AUTHOR}

Dr. Muzhir Shaban Al-Ani is with the Department of Management Information Systems o the Amman Arab University of Graduate Studies, Amman, Jordan, 11953

Manuscript received 09 September 2008. Published as submitted by the author. 